Article

\title{
Self-Inductance of the Circular Coils of the Rectangular Cross-Section with the Radial and Azimuthal Current Densities
}

\author{
Slobodan Babic ${ }^{1, *}$ and Cevdet Akyel ${ }^{2}$ \\ 1 Independent Researcher, 53 Berlioz, 101, Montréal, QC H3E 1N2, Canada \\ 2 École Polytechnique, C.P. 6079 Centre-Ville, Montréal, QC H3C 3A7, Canada; cevdet.akyel@polymtl.ca \\ * Correspondence: slobobob@yahoo.com
}

Received: 15 June 2020; Accepted: 6 July 2020; Published: 13 July 2020

check for updates

\begin{abstract}
In this paper, we give new formulas for calculating the self-inductance for circular coils of the rectangular cross-sections with the radial and the azimuthal current densities. These formulas are given by the single integration of the elementary functions which are integrable on the interval of the integration. From these new expressions, we can obtain the special cases for the self-inductance of the thin-disk pancake and the thin-wall solenoids that confirm the validity of this approach. For the asymptotic cases, the new formula for the self-inductance of the thin-wall solenoid is obtained for the first time in the literature. In this paper, we do not use special functions such as the elliptical integrals of the first, second and third kind, nor Struve and Bessel functions because that is very tedious work. The results of this work are compared with already different known methods and all results are in excellent agreement. We consider this approach novel because of its simplicity in the self-inductance calculation of the previously-mentioned configurations.
\end{abstract}

Keywords: self-inductance; radial current; azimuthal current; thick coils; disk coils; solenoids

\section{Introduction}

Several monographs and papers are devoted to calculating the self and the mutual inductance for the circular coils of the rectangular cross-section with the azimuthal current density [1-18]. The conventional coils used in many applications such as all ranges of transformers, generators, motors, current reactors, magnetic resonance applications, antennas, coil guns, medical electronic devices, superconducting magnets, tokamaks, electronic and printed circuit board design, plasma science, etc., are very well-known. Today, with the availability of powerful and general numerical methods, such as finite element method (FEM) and boundary element method (BEM), it is possible to accurately and rapidly calculate the self and mutual inductance of almost any practical 3D geometric arrangement of conductors. However, in many circumstances, there is still an interest to address this problem using analytic and semi-analytic methods because they considerably simplify the mathematical procedures, and often lead to a significant reduction of the computational effort.

The analytical and semi-analytical methods have been used where these important electromagnetic quantities are obtained in the form of the simple, double and triple integrals, the elliptic integrals, the converge series, the Bessel functions, Struve functions [1-18]. There are circular coils of the rectangular cross-section with the radial current density which are interesting from an engineering aspect. These coils are the well-known Bitter coils [19-25] which supply extremely high magnetic fields up to $45 \mathrm{~T}$ [21].

In this paper, our goal is to analytically solve the four integrals in the basic formulas for the self-inductance of the circular coils of the rectangular cross-section with radial and the azimuthal 
current densities, namely $L_{R}$ (radial current) and $L_{A}$ (azimuthal current), respectively. We obtained all results of these four integrations in the form of elementary analytical functions. By the single integration of these expressions, we obtained the simplest formulas for calculating the self-inductance of $L_{R}$ and $L_{A}$ without using the special functions. All expressions are arranged in a suitable form for the numerical integration where the possible singularities are treated at the proper manner. Numerous tests are made in MATLAB and Mathematica programing which show that the numerical integration given in Mathematica programming can be used for any range of parameters (very small or very large values of $\alpha=\frac{R_{2}}{R_{1}}$ and $b=\frac{l}{R_{1}}$ ), where $R_{1}$ and $R_{2}$ are the inner and outer radius of the coil and $l$ is its hight [26,27]. Many examples confirm the validity of the presented method. With the presented method all possible cases for the circular coils with a finite cross-section or negligible cross-section (thin coils and circular filamentary coils) are covered.

\section{Basic Expressions}

Let us consider the circular coil of the rectangular cross-section, as shown in Figure 1. Here, $R_{1}$ is the inner radius (in $\mathrm{m}$ ), $R_{2}$ is the outer radius (in $\mathrm{m}$ ), $l$ is the height of the coil (in $\mathrm{m}$ ), $I$ is the current in the coil (in A), $J_{R}$ is the radial current density (in A/m $\mathrm{m}^{2}$ ), $J_{A}$ is the azimuthal current density (in $\left.\mathrm{A} / \mathrm{m}^{2}\right), r_{1}, r_{2}$ are the coordinates which determine any radial position inside the coil (in $\mathrm{m}$ ), $z_{1}, z_{2}$ are the coordinates which determine any axial position inside the coil (in $\mathrm{m}$ ), $N$ is the number of turns, $\mu_{0}=4 \pi \cdot 10^{-7} \mu \mathrm{H} / \mathrm{m}$ is the permeability of the free space.

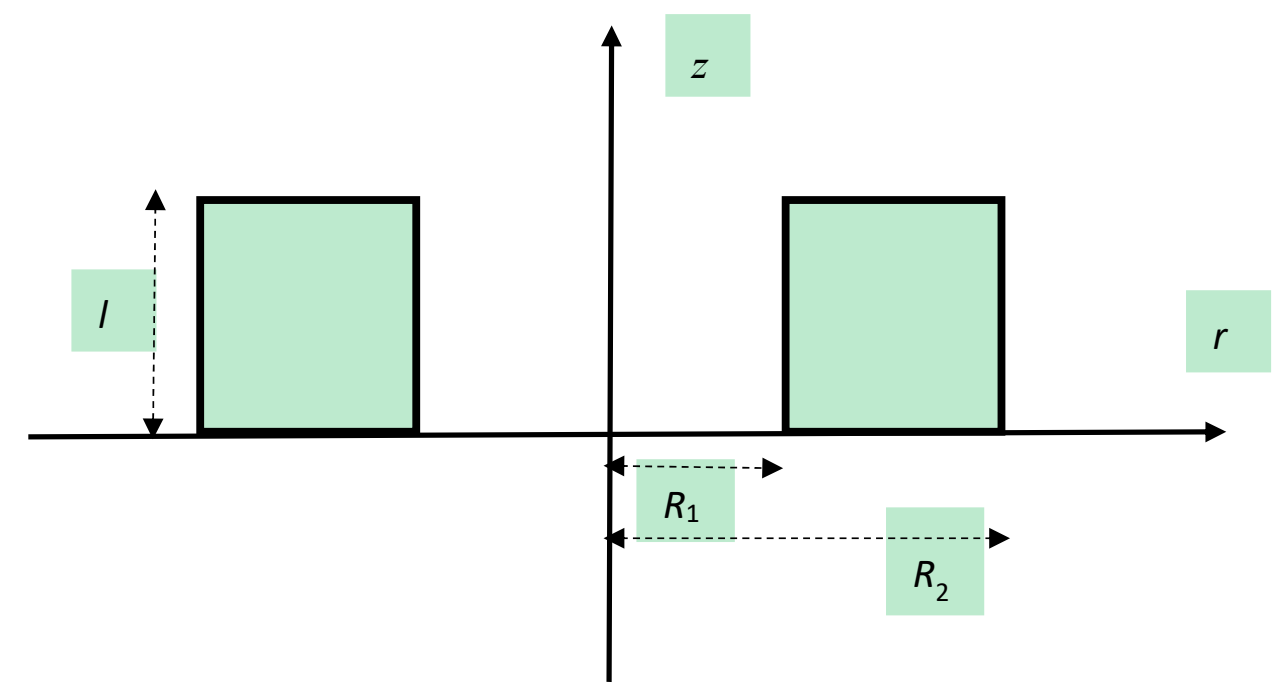

Figure 1. Circular thick coil of the rectangular cross-section.

\subsection{Radial Current}

The radial current density and the corresponding self-inductance of the coil of the rectangular cross-section are given by [15-20]:

$$
\begin{gathered}
J_{R}=\frac{N I}{l \ln \frac{R_{2}}{R_{1}}} \cdot \frac{1}{r}, \\
L_{R}=\frac{\mu_{0} N^{2}}{l^{2} \ln ^{2} \frac{R_{2}}{R_{1}}} \int_{0}^{l} \int_{0}^{l} \int_{R_{1}}^{R_{2}} \int_{R_{1}}^{R_{2}} \int_{0}^{\pi} \frac{\cos (\theta) d z_{1} d z_{2} d r_{1} d r_{2} d \theta}{R_{12}},
\end{gathered}
$$

where,

$$
R_{12}=\sqrt{r_{1}^{2}+r_{2}^{2}-2 r_{1} r_{2} \cos (\theta)+\left(z_{2}-z_{1}\right)^{2}}
$$




\subsection{Azimuthal Current}

The azimuthal current density and the corresponding self-inductance of the coil of the rectangular cross-section are given by [15-20]:

$$
\begin{gathered}
J_{A}=\frac{N I}{l\left(R_{2}-R_{1}\right)}, \\
L_{A}=\frac{\mu_{0} N^{2}}{l^{2}\left(R_{2}-R_{1}\right)^{2}} \int_{0}^{l} \int_{0}^{l} \int_{R_{1}}^{R_{2}} \int_{R_{1}}^{R_{2}} \int_{0}^{\pi} \frac{\cos (\theta) d z_{1} d z_{2} r_{1} d r_{1} r_{2} d r_{2} d \theta}{R_{12}} .
\end{gathered}
$$

\section{Calculation Method}

\subsection{The Self-Inductance $L_{R}$ Caused by the Radial Current Density}

Introducing the substitution $r_{1}=x R_{1}, r_{2}=y R_{1}, z_{1}=v R_{1}, z_{2}=z R_{1}, t=v-z, l=b R_{1}$, $R_{2}=\alpha R_{1}, \theta=\pi-2 \beta$, in Equation (2) and making the first four integrations in order to the variables $z_{2}, z_{1}, r_{2}$ and $r_{1}$, or $y, x, v$ and $z$, (Appendix A) we obtained the self-inductance $L_{R}$ in the following form:

$$
L_{R}=-\frac{2 \mu_{0} N^{2} R_{1}}{b^{2} \ln ^{2}(\alpha)} \sum_{n=1}^{11} \int_{0}^{\frac{\pi}{2}} \cos (2 \beta) T_{n} d \beta,
$$

where

$$
\begin{gathered}
T_{1}=\frac{b^{3}}{3 \sin (2 \beta)}\left[2 \arctan (q)-\arctan \left(q_{1}\right)-\arctan \left(q_{2}\right)\right], \\
T_{2}=\frac{8}{3}\left(\alpha^{3}+1\right) \cos ^{3}(\beta)-\frac{4}{3} \cos ^{2}(\beta)\left(\alpha^{2} r_{2}+r_{1}\right)+\frac{2}{3}\left[\left(\alpha^{2}+1\right) \cos (2 \beta) 2 \alpha\right]\left(r-r_{0}\right), \\
T_{3}=4 b \alpha^{2} \cos ^{2}(\beta) \operatorname{arsinh}\left[\frac{b}{2 \alpha \cos (\beta)}\right]+4 b \cos ^{2}(\beta) \operatorname{arsinh}\left[\frac{b}{2 \cos (\beta)}\right] \\
-2 b\left[\left(\alpha^{2}+1\right) \cos (2 \beta)+2 \alpha\right] \operatorname{arsinh}\left(\frac{b}{r_{0}}\right), \\
T_{4}=2 b^{2}\left[\alpha \operatorname{arsinh}\left(v_{22}\right)+\operatorname{arsinh}\left(v_{11}\right)\right], \\
T_{5}=-2 b^{2}\left[\alpha \operatorname{arsinh}\left(v_{2}\right)+\operatorname{arsinh}\left(v_{1}\right)\right], \\
T_{6}=-2 b \sin (2 \beta)\left[\alpha^{2} \arctan \left(p_{22}\right)+\arctan \left(p_{11}\right)\right] \\
T_{7}=2 b \sin (2 \beta)\left[\alpha^{2} \arctan \left(p_{2}\right)+\arctan \left(p_{1}\right)\right] \\
T_{8}=\frac{\alpha^{3}}{3} \sin ^{2}(2 \beta) \ln \left(\frac{m_{2}+1}{m_{2}-1}\right)+\frac{1}{3} \sin ^{2}(2 \beta) \ln \left(\frac{m_{1}+1}{m_{1}-1}\right), \\
T_{9}=-\frac{\alpha^{3}}{3} \sin ^{2}(2 \beta) \ln \left(\frac{m_{20}+1}{m_{20}-1}\right)-\frac{1}{3} \sin ^{2}(2 \beta) \ln \left(\frac{m_{10}+1}{m_{10}-1}\right), \\
T_{10}=-\frac{\alpha^{3}}{3} \sin ^{2}(2 \beta) \ln \left(\frac{m_{22}+1}{m_{22}-1}\right)-\frac{1}{3} \sin ^{2}(2 \beta) \ln \left(\frac{m_{11}+1}{m_{11}-1}\right), \\
T_{11}=\frac{2\left(\alpha^{3}+1\right)}{3} \sin ^{2}(2 \beta) \ln \left[\frac{\cos (\beta / 2)}{\cos (\beta / 2)}\right]
\end{gathered}
$$


where

$$
\begin{gathered}
r=\sqrt{b^{2}+\alpha^{2}+1+2 \alpha \cos (2 \beta)}, \quad r_{0}=\sqrt{\alpha^{2}+1+2 \alpha \cos (2 \beta)}, \quad r_{1}=\sqrt{b^{2}+4 \cos ^{2}(\beta)}, \\
r_{2}=\sqrt{b^{2}+4 \alpha^{2} \cos ^{2}(\beta)}, \quad r_{01}=\sqrt{4 \cos ^{2}(\beta)}=2 \cos (\beta), \quad r_{02}=\sqrt{4 \alpha^{2} \cos ^{2}(\beta)}=2 \alpha \cos (\beta), \\
q=\frac{\alpha \sin ^{2}(2 \beta)-b^{2} \cos (2 \beta)}{b \sin (2 \beta) r}, \quad q_{1}=\frac{\alpha^{2} \sin ^{2}(2 \beta)-b^{2} \cos (2 \beta)}{b \sin (2 \beta) r_{2}}, \\
q_{2}=\frac{\sin ^{2}(2 \beta)-b^{2} \cos (2 \beta)}{b \sin (2 \beta) r_{1}}, \quad v_{1}=\frac{\alpha+\cos (2 \beta)}{\sqrt{b^{2}+\sin ^{2}(2 \beta)}}, \quad v_{2}=\frac{1+\alpha \cos (2 \beta)}{\sqrt{b^{2}+\alpha^{2} \sin ^{2}(2 \beta)}}, \\
v_{11}=\frac{1+\cos (2 \beta)}{\sqrt{b^{2}+\sin ^{2}(2 \beta)}}, \quad v_{22}=\frac{\alpha+\alpha \cos (2 \beta)}{\sqrt{b^{2}+\alpha^{2} \sin ^{2}(2 \beta)}}, \quad p_{1}=\frac{b[\alpha+\cos (2 \beta)]}{\sin (2 \beta) r}, \\
p_{2}=\frac{b[1+\alpha \cos (2 \beta)]}{\alpha \sin (2 \beta) r}, \quad p_{11}=\frac{b[1+\cos (2 \beta)]}{\sin (2 \beta) r_{1}}, \quad p_{22}=\frac{b[\alpha+\alpha \cos (2 \beta)]}{\alpha \sin (2 \beta) r_{2}}, \\
m_{1}=\frac{r}{\alpha+\cos (2 \beta)}, \quad m_{2}=\frac{r}{1+\alpha \cos (2 \beta)}, \quad m_{10}=\frac{r_{0}}{\alpha+\cos (2 \beta)}, \quad m_{20}=\frac{r_{0}}{1+\alpha \cos (2 \beta)}, \\
m_{11}=\frac{r_{1}}{1+\cos (2 \beta)}, \quad m_{22}=\frac{r_{2}}{\alpha+\alpha \cos (2 \beta)} .
\end{gathered}
$$

Expressions for $T_{n}, n=1,2, \ldots, 11$, are given as in the Addendum where we calculate the self-inductance for the coil of the rectangular cross-section with radial current in Example 2 in the Addendum.

Thus, the new formula for the self-inductance of the circular coil with the rectangular cross-section and the radial current density can be obtained by Equation (5) using the simple integration of the previous elementary functions. In this paper, we use the Gaussian numerical integration in MATLAB programming and the numerical integration by default in Mathematica programing.

The special case of Equation (5) is the self-inductance of the thin-disk coil with a radial current [23]. This self-inductance can be obtained from Equation (5) by finding the limit when $b \rightarrow 0$, or doing three integrations such as in [23].

The self-inductance $L_{R \text {-disk }}$ is obtained in the analytical form as follows:

$$
L_{R-\text { disk }}=\frac{4 \mu_{0} N^{2} R_{1}(\alpha+1)}{\ln ^{2} \alpha}\left[E\left(k_{0}\right)-1\right],
$$

where

$$
k_{0}^{2}=\frac{4 \alpha}{(\alpha+1)^{2}},
$$

and $E\left(k_{0}\right)$ is the elliptic integral of the second kind [23].

\subsection{The Self-Inductance $L_{A}$ Caused by the Azimuthal Current Density}

Introducing the substitution $r_{1}=x R_{1}, r_{2}=y R_{1}, z_{1}=v R_{1}, z_{2}=z R_{1}, t=v-z, l=b R_{1}$, $R_{2}=\alpha R_{1}, \theta=\pi-2 \beta$, in Equation (2) and making the first four integrations in order to the variables $z_{2}, z_{1}, r_{2}$ and $r_{1}$, or $y, x, v$ and $z$, (Appendix B) we obtained the self-inductance $L_{A}$ in the following form

$$
L_{A}=-\frac{\mu_{0} N^{2} R_{1}}{15 b^{2}(\alpha-1)^{2}} \sum_{n=1}^{6} \int_{0}^{\frac{\pi}{2}} \cos (2 \beta) S_{n} d \beta,
$$


where

$$
\begin{gathered}
S_{1}=\frac{b^{4}}{\sin ^{2}(2 \beta)}\left[r_{2}-\frac{b \cos (2 \beta)}{\sin (2 \beta)} \arctan \left(q_{2}\right)\right]+\frac{b^{4}}{\sin ^{2}(2 \beta)}\left[r_{1}-\frac{b \cos (2 \beta)}{\sin (2 \beta)} \arctan \left(q_{1}\right)\right] \\
-\frac{2 b^{4}}{\sin ^{2}(2 \beta)}\left[r-\frac{b \cos (2 \beta)}{\sin (2 \beta)} \arctan (q)\right], \\
S_{2}=9 a^{2} b^{2} r_{2}+9 b^{2} r_{1}-9\left(a^{2}+1\right) b^{2} r+2\left[6 a^{4} \cos ^{2}(2 \beta)-2 a^{4} \cos (2 \beta)-8 a^{4}\right]\left(r_{2}-r_{02}\right) \\
+2\left[6 \cos ^{2}(2 \beta)-2 \cos (2 \beta)-8\right]\left(r_{1}-r_{01}\right)-4\left[3\left(a^{4}+1\right) \cos ^{2}(2 \beta)-\alpha\left(a^{2}+1\right) \cos (2 \beta)\right. \\
\left.-2\left(\alpha^{2}+1\right)^{2}\right]\left(r-r_{0}\right), \\
S_{4}=30 b \sin (2 \beta) \cos (2 \beta)\left[a^{4} \arctan \left(p_{22}\right)+\arctan \left(p_{11}\right)-a^{4} \arctan \left(p_{2}\right)-\arctan \left(p_{1}\right)\right], \\
15 b\left\{\alpha^{4} \sin ^{2}(2 \beta) \ln \left(\frac{r_{2}+b}{r_{2}-b}\right)+\sin ^{2}(2 \beta) \ln \left(\frac{r_{1}+b}{r_{1}-b}\right)-\frac{1}{2}\left[\left(\alpha^{2}+1\right)^{2}-2\left(\alpha^{4}+1\right) \cos { }^{2}(2 \beta)\right] \ln \left(\frac{r+b}{r-b}\right)\right\}, \\
S_{5}=12 \cos (2 \beta) \sin ^{2}(2 \beta)\left\{\alpha^{5} \ln \left[r_{0}+1+\alpha \cos (2 \beta)\right]+\ln \left[r_{0}+\alpha+\cos (2 \beta)\right]\right. \\
\left.-\left(\alpha^{5}+1\right) \ln \left[4 \cos (\beta) \cos ^{2}(\beta / 2)\right]-\alpha^{5} \ln (\alpha)\right\}, \\
S_{6}=4 \cos (2 \beta)\left[5 b^{2}-3 \sin ^{2}(2 \beta)\right] \ln \left[\frac{r+\alpha+\cos (2 \beta)}{r_{1}+1+\cos (2 \beta)}\right] \\
+4 \alpha^{3} \cos (2 \beta)\left[5 b^{2}-3 \alpha^{2} \sin ^{2}(2 \beta)\right] \ln \left[\frac{r+1+\alpha \cos (2 \beta)}{r_{2}+\alpha+\alpha \cos (2 \beta)}\right] .
\end{gathered}
$$

Expressions for $S_{n}, n=1,2, \ldots, 6$, are given as in the Addendum where we calculate the self-inductance for the coil of the rectangular cross-section with azimuthal current in Example 4 $\alpha=3, b=2)$ in the Addendum.

Thus, the self-inductance of the circular coil of the rectangular cross-section with the azimuthal current density can be obtained by Equation (7) using simple integration of the previous elementary functions.

The special case of this calculation is the self-inductance of the thin-disk coil (pancake) with the azimuthal current $[26,27]$. This self-inductance can be obtained from Equation (7) finding the limit when $\alpha \rightarrow 0$ or doing the three integration such as in [26].

The self-inductance $L_{A \text {-disk }}$ is obtained in the analytical form as follows:

$$
L_{A-\text { disk }}=\frac{2 \mu_{0} N^{2} R_{1}}{3(\alpha-1)^{2}} V
$$

where

$$
\begin{gathered}
V=\alpha(\alpha+1) E\left(k_{0}\right)+\left(\alpha^{3}+1\right)(2 G-1)+\frac{\pi}{2} \ln \frac{k_{0}}{2}+S_{10}+\left(\alpha^{3}-1\right) S_{2}, \\
k_{0}^{2}=\frac{4 \alpha}{(\alpha+1)^{2}}, \\
S_{1}=\int_{0}^{\frac{\pi}{2}} \ln [1+\Delta] d \beta, \quad S_{2}=\int_{0}^{\frac{\pi}{2}} \ln \left[\sqrt{1-k_{0}^{2}}+\Delta\right] d \beta, \quad \Delta=\sqrt{1-k_{0}^{2} \sin ^{2}(\beta)},
\end{gathered}
$$

and $G=0.915965594 \ldots$ is the Catalan's constant $[26,27]$.

The self-inductance is obtained as the combinations of the elementary functions, the elliptical integral of the second kind [28-30], and the single integrals (the semi-analytical solution).

In [27], the new expression for $V$ is given by:

$$
V=\alpha(\alpha+1) E\left(k_{0}\right)+\left(\alpha^{3}+1\right)(2 G-1)-\frac{\pi}{2} \ln 2-S_{10}-\alpha^{3} S_{20},
$$




$$
\begin{aligned}
& S_{10}=\int_{0}^{\frac{\pi}{2}} \ln \left[\alpha+\cos (2 \beta)+\sqrt{\alpha^{2}+2 \alpha \cos (2 \beta)+1}\right] d \beta, \\
& S_{20}=\frac{1}{2} \int_{0}^{\frac{\pi}{2}} \ln \frac{\alpha \cos (\beta)+1+\sqrt{\alpha^{2}+2 \alpha \cos (\beta)+1}}{\alpha \sin (\beta)-1+\sqrt{\alpha^{2}-2 \alpha \sin (\beta)+1}} d \beta .
\end{aligned}
$$

This expression is also very friendly for the numerical integration in Equation (9).

For the full disk $\left(R_{1}=0, R_{2}=R\right)$, the self-inductance is

$$
L_{A-\text { full-disk }}=\frac{2 \mu_{0} N^{2} R_{1}}{3}[2 G-1] .
$$

This formula can be found in $[10,27,31]$.

There is also one special case when $R_{1} \rightarrow R_{2} \rightarrow R$ (thin-wall solenoid of radius $R$ and hight $l$ ). Finding the limit in Equation (7) or solving the three integrals in [32], we obtain the well-known Lorentz's formula (1879),

$$
L_{A-\text { wall-solen }}=\frac{2 \mu_{0} N^{2} R^{2}}{3 l}\left[\frac{l}{k R} K(k)-\frac{l^{2}-4 R^{2}}{k l R} E(k)-4 \frac{R}{l}\right],
$$

where

$$
k^{2}=\frac{4 R^{2}}{4 R^{2}+l^{2}},
$$

and $K(k), E(k)$ are the elliptic integrals of the first and second kind [32].

From previous formulas for $L_{R}$ and $L_{A}$ it is obvious that they have similar terms and all expressions are elementary functions that are very friendly for single numerical integration. The special cases are obtained as the analytical and semi-analytical expressions for these important electromagnetic quantities (6)-(12).

\subsection{Asymptotic Behaviors of Disk Coils and Thin-Wall Solenoids}

At first, we analyze the disk coil.

For $R_{1}=R_{2}$ we have a well-known singular case which gives

$$
L=\infty .
$$

For $R_{1} \rightarrow R_{2} \rightarrow R$ (inner radius tends toward the outer radius), this case leads to the well-known formula [31]

$$
L=\mu_{0} N^{2} R\left[\ln \frac{8 R}{a}-2+Y\right] .
$$

where $R$ is the turn radius, $a$ is the radius of the circular wire from which the turn is constructed. If the current flows only on the wire surface (due to the skin effect) $Y=0$, and the current flow is homogeneous in the wire, then $Y=0.25$.

For $R_{1} \approx R_{2}$ (the case of a logarithmic singularity), Conway [31] gives the analogous formula

$$
L=\mu_{0} N^{2} R_{2}\left[\ln \frac{8 R_{2}}{R_{2}-R_{1}}-\frac{1}{2}\right] .
$$

From Kirchhoff's formula for the self-inductance of a circular ring of the radius $R$ and the circular section of the radius $a$ with one turn [13], we have

$$
L=\mu_{0} R\left[\ln \frac{8 R}{a}-1.75\right] .
$$


Luo, Y. and Chan, B. [13] obtained, for this asymptotic case

$$
L=\mu_{0} \frac{\left(R_{2}+R_{1}\right)}{R_{1}}\left[\ln \frac{4\left(R_{2}+R_{1}\right)}{\left(R_{2}-R_{1}\right)}-0.5\right](\mathrm{H} / \mathrm{m}) .
$$

The asymptotic case for the thin-wall solenoid can be calculated from [13]

$$
L=\mu_{0}\left[\ln \frac{4 R}{h}-0.5\right](\mathrm{H} / \mathrm{m})
$$

where $R$ is the wall solenoid's radius and $2 h$ is its hight.

From this approach, the self-inductance of a thin-wall solenoid in the asymptotic case is obtained for the first time in the literature.

Let us put in Equation (12)

$$
b=\frac{l}{2 R}, \quad k^{2}=\frac{1}{1+b^{2}},
$$

so that the self-inductance of the thin-wall solenoid is

$$
L_{A-\text { wall-solen }}=\frac{2 \mu_{0} N^{2} R}{3 k}\left[K(k)+\frac{1-b^{2}}{b^{2}} E(k)-\frac{k}{b^{2}}\right] .
$$

To find the self-inductance of thin-wall solenoid for $b \rightarrow 0$, the asymptotic behavior of $K(k)$ and $K(k)$ near the singularity at $k=1$ are given by the following expression [33]:

$$
\begin{gathered}
K(k) \sim \ln \frac{4}{k^{\prime}}=\ln \frac{4}{\sqrt{1-k^{2}}}=\ln \frac{4}{b}+\ln \sqrt{1+b^{2}} \\
E(k) \sim 1+\frac{1}{2}\left(1-k^{2}\right)\left[\ln \frac{4}{k^{\prime}}-0.5\right]=1+\frac{b^{2}}{2\left(1+b^{2}\right)}\left[\ln \frac{4}{k^{\prime}}-0.5\right] .
\end{gathered}
$$

The approximations (20) and (21) are the first terms of the convergent series [28-30,33]. We calculate the normalized self-inductance of the extremely short-wall solenoid $(b \rightarrow 0)$ as

$$
L_{N}=\frac{L_{A-\text { wall-solen }}}{N^{2} R}
$$

From Equations (19)-(22) we finally have:

$$
L_{N}=\frac{\mu_{0}}{3}\left[\frac{\left(3+b^{2}\right)}{\sqrt{1+b^{2}}} \ln \frac{4 \sqrt{1+b^{2}}}{b}+\frac{2\left[\sqrt{1+b^{2}}\left(1-b^{2}\right)-1\right]}{b^{2}}-\frac{\left(1-b^{2}\right)}{2 \sqrt{1+b^{2}}}\right]
$$

For $b$ extremely near at zero, we find using the 1'Hospital's Rule from Equation (23) that the first term tends to $\ln \frac{4}{b}$, the second to -1 and the third to -0.5 . Finally, the self-inductance from this range of the parameter $b$ is

$$
L_{N}=\mu_{0}\left[\ln \frac{4}{b}-\frac{1}{2}\right](\mathrm{H} / \mathrm{m}) .
$$

This formula has been obtained by the ansatz in [13].

To our knowledge, the formula (23) appears for the first time in the literature.

Thus, we cover all possible cases with the new formulas and the already well-known or the improved formulas in the calculation of the self-inductance of the previously mentioned circular coils. 


\section{Numerical Validation}

To verify the validity of the new formulas for the self-inductances $L_{R}$ and $L_{A}$ we applied the following set of examples. The special cases are discussed. We compared the results of the presented approach with those known in the literature.

Example 1. Calculate the self-inductance of the thick Bitter circular coil of a rectangular cross-section. The coil dimensions and the number of turns is as follows:

$$
R_{1}=1 \mathrm{~m}, R_{2}=2 \mathrm{~m}, l=2 \mathrm{~m}, N=100 .
$$

Applying the new formula (5), the self-inductance is

$$
L_{R}=17.815333 \mathrm{mH} .
$$

By using Conway's method [20], the self-inductance is

$$
L_{\text {Conway }}=17.815333 \mathrm{mH} \text {. }
$$

The results are in excellent agreement.

Example 2. Calculate the self-inductance of the thick Bitter circular coil of a rectangular cross-section. The coil dimensions and the number of turns is as follows:

$$
R_{1}=0.025 \mathrm{~m}, R_{2}=0.035 \mathrm{~m}, l=0.04 \mathrm{~m}, N=100 .
$$

Using (5) we obtain

$$
L_{R}=0.4383980 \mathrm{mH} .
$$

By using Ren's method [21,22], the self-inductance is

$$
L_{\text {Ren }}=0.4383978 \mathrm{mH} .
$$

This self-inductance is obtained by double integration.

Using the software ANSYS (FEM) [20,21] the self-inductance is

$$
L_{\text {Ren }}=0.44528 \mathrm{mH} \text {. }
$$

All results are in good agreement.

Example 3. Calculate the self-inductance of the thin Bitter disk (pancake) [23]. The coil dimensions and the number of turns is as follows:

$$
R_{1}=1 \mathrm{~m}, R_{2}=2 \mathrm{~m}, N=1000 .
$$

The formula (6) gives

$$
L_{R-\text { disk }}=3.56991288673 \mathrm{H} \text {. }
$$

Example 4. In Table 1 we compare the results obtained by this work (7), [9,13]. 
Table 1. The accuracy and the computational time for the self-inductance calculation $[9,13], L_{A}$.

\begin{tabular}{|c|c|c|c|c|}
\hline$\alpha$ & $b$ & $L_{\text {Luo }}(\mu \mathrm{H} / \mathrm{m})[13]$ & $L_{\text {Kajikawa }}(\mu \mathrm{H} / \mathrm{m})[9]$ & $L_{\text {This Work }}(\mu \mathrm{H} / \mathrm{m})$ \\
\hline 1.5 & 0.5 & 2.8693036 & 2.8693 & 2.8693035 \\
\hline 3.0 & 2.0 & 2.5330065 & 2.533 & 2.5330065 \\
\hline 4.0 & 6.0 & 1.9012958 & 1.9012 & 1.9012958 \\
\hline 7.0 & 12.0 & 2.4472979 & 2.4473 & 2.4472979 \\
\hline 9.0 & 8.0 & 4.2674018 & 4.2661 & 4.2674018 \\
\hline
\end{tabular}

In these calculations, we take $R_{1}=1 \mathrm{~m}$ and $\alpha=\frac{R_{2}}{R_{1}}, b=\frac{2 l}{R_{1}}$ (see Table 1 ).

From Table 1, we can see the excellent agreement between this work (7) and [13], and good agreement with [9].

Example 5. Let us compare the results of the formula (7) by those obtained using [9,13], as shown in Table 2.

Table 2. The comparison with Luo and Kajikawa formulas.

\begin{tabular}{lllll}
\hline \multicolumn{1}{r}{$\boldsymbol{\alpha}$} & $\boldsymbol{b}$ & \multicolumn{1}{c}{$\boldsymbol{L}_{\text {Luo }}(\mu \mathrm{H} / \mathrm{m})[13]$} & \multicolumn{1}{c}{$\boldsymbol{L}_{\text {Kajikawa }}(\mu \mathrm{H} / \mathrm{m})[9]$} & $\boldsymbol{L}_{\text {This Work }}(\mu \mathrm{H} / \mathrm{m})$ \\
\hline 1.2 & 20.0 & 0.2142821 & 0.21428 & 0.2142821 \\
5.0 & 20.0 & 1.0456844 & 1.0457 & 1.0456844 \\
20.0 & 20.0 & 7.8764442 & 7.867 & 7.8764442 \\
40.0 & 20.0 & 19.950453 & 19.951 & 19.950453 \\
1.2 & 2.0 & 1.4613306 & 1.4618 & 1.4613306 \\
5.0 & 2.0 & 3.8343885 & 3.8343 & 3.8343885 \\
20.0 & 2.0 & 14.120116 & 14.112 & 14.120116 \\
40.0 & 2.0 & 28.015984 & 27.992 & 28.015984 \\
1.2 & 0.2 & 3.5880363 & 3.588 & 3.5880363 \\
5.0 & 0.2 & 5.0682989 & 5.0681 & 5.0682989 \\
20.0 & 0.2 & 15.288175 & 15.28 & 15.288175 \\
40.0 & 0.2 & 29.185174 & 29.161 & 29.185174 \\
\hline
\end{tabular}

In these calculations we take $R_{1}=1 \mathrm{~m}$ and $\alpha=\frac{R_{2}}{R_{1}}, b=\frac{2 l}{R_{1}}$. In [13], the hight of the coil is $2 h$ and $b=\frac{h}{R_{1}}$. In [9], the same parameters were used as in this paper.

From Table 2, one can see that the results of this work and those in [13] are in an excellent agreement and in particularly good agreement whit Kajikawa results [9] where the number of significant figures in the calculation was about three.

Example 6. In this example, we compare the results of the self-inductance (7) with the self-inductance obtained by Bessel functions [10] (Table 3).

Table 3. The comparison with Conway's formula and Kajikawa's formula.

\begin{tabular}{|c|c|c|c|c|}
\hline$\alpha$ & $b$ & $L_{\text {Conway }}(\mu \mathrm{H} / \mathrm{m})[10]$ & $L_{\text {Kajikawa }}(\mu \mathrm{H} / \mathrm{m})[9]$ & $L_{\text {This Work }}(\mu \mathrm{H} / \mathrm{m})$ \\
\hline 1.5 & 0.5 & 2.8693035 & 2.8693 & 2.8693035 \\
\hline 3.0 & 2.0 & 2.5330065 & 2.533 & 2.5330065 \\
\hline 4.0 & 6.0 & 1.9012858 & 1.9012 & 1.9012858 \\
\hline 7.0 & 12.0 & 2.4472979 & 2.4473 & 2.4472979 \\
\hline 9.0 & 8.0 & 4.2676018 & 4.2661 & 4.2676018 \\
\hline
\end{tabular}

There is an excellent agreement between Conway's method and this work and particularly good agreement by Kajikawa's method, as seen from Table 3. 
Example 7. In this example, we calculate the normalized self-inductance of the thin-disk coil (pancake) regarding the inner radius and the number of turns for the different shape factor $\alpha=\frac{R_{2}}{R_{1}}$ (Table 4$)$. We compare the results of Formulas (8)-(10) with [4,31].

Table 4. Comparison of Computational Accuracy for the different shape factor $\alpha$.

\begin{tabular}{lllll}
\hline \multicolumn{1}{c}{$\alpha$} & $\boldsymbol{L}_{\text {Spielrein }}(\mu \mathrm{H} / \mathrm{m})$ & $\boldsymbol{L}_{\text {Conway }}(\mu \mathrm{H} / \mathrm{m})$ & $\boldsymbol{L}_{(8),(9)}(\mu \mathrm{H} / \mathrm{m})$ & $\boldsymbol{L}_{(8),(10)}(\mu \mathrm{H} / \mathrm{m})$ \\
\hline 5.0 & 36.282205 & 36.282205 & 36.282205 & 36.282205 \\
10.0 & 8.5558079 & 8.5558079 & 8.5558079 & 8.5558079 \\
3.0 & 4.1202479 & 4.1202478 & 4.1202478 & 4.1202478 \\
1.5 & 3.9375566 & 3.9375570 & 3.9375569 & 3.9375569 \\
1.1 & 5.1875898 & 5.1875898 & 5.1875898 & 5.1875898 \\
1.01 & 7.8169836 & 7.8169836 & 7.8169836 & 7.8169836 \\
1.001 & 10.671287 & 10.671287 & 10.671287 & 10.671287 \\
1.00001 & 16.452442 & 16.452442 & 16.452442 & 16.452442 \\
1.000001 & 19.345878 & 19.345878 & 19.345878 & 19.345878 \\
1.0000001 & 22.239382 & 22.239382 & 22.239382 & 22.239382 \\
\hline
\end{tabular}

From presented results obtained by formulas (8)-(10), and from Spielrein's and Conway's approaches, we can see that all of them are in an excellent agreement. There is negligible disagreement with Spielrein's approach where the self-inductance was calculated by a series which does not converge quickly.

Example 8. In this example, the self-inductance of the disk is calculated when $\alpha$ is remarkably close to 1 (Table 5) until the extreme case $\alpha=1$ for which the self-inductance is $\infty$.

Table 5. Comparison of computational accuracy for the shape factor $\alpha$ close to 1 .

\begin{tabular}{lll}
\hline & \multicolumn{1}{c}{$L_{\text {Conway }}(\mu \mathrm{H} / \mathrm{m})$} & \multicolumn{1}{c}{$L_{(8),(10)}(\mu \mathrm{H} / \mathrm{m})$} \\
\hline $10^{-1}$ & 5.1875898 & 5.1875898 \\
$10^{-2}$ & 7.8169836 & 7.8169836 \\
$10^{-3}$ & 10.671287 & 10.671287 \\
$10^{-4}$ & 19.345878 & 19.345878 \\
$10^{-8}$ & 25.132895 & 25.132895 \\
$10^{-10}$ & 25.132895 & 25.132895 \\
$10^{-12}$ & 36.706950 & 36.706950 \\
$10^{-15}$ & 45.387491 & 45.387491 \\
$10^{-16}$ & 45.387491 & 45.387491 \\
\hline
\end{tabular}

From Table 5, where $\alpha$ is extremely close to 1, expressions (8)-(10) give the same results as Conway's approach.

Example 9. In this example, we compare the results for the self-inductance (23) by those obtained in [13] (Equations (27) and (46)) and in [9].

As one can see from Table 6, all results are in an excellent agreement. It is obvious that the formula (23) gives more precise results for the range of $10^{-6} \leq b \leq 10^{-1}$ then formula (46) in [13] and for $b \leq 10^{-6}$ the formula (24) is the same as the formula (46) in [13]. Kajikawa's method gives particularly good results for $10^{-3} \leq b \leq 10^{-1}$. With this calculation, we confirm the validity of the newly developed formula (23) which also leads to formula (24). 
Table 6. Comparison of Computational Accuracy for the different shape factor $b$.

\begin{tabular}{lllll}
\hline \multicolumn{1}{c}{$\boldsymbol{b}$} & $\boldsymbol{L}_{\text {Luo(27) }}$ or $L_{(12)}(\mu \mathrm{H} / \mathrm{m})$ & $L_{\text {Luo(46) }}(\mu \mathrm{H} / \mathrm{m})$ & $L_{\text {This Work(23) }}(\mu \mathrm{H} / \mathrm{m})$ & \multicolumn{1}{c}{$\boldsymbol{L}[9](\mu \mathrm{H} / \mathrm{m})$} \\
\hline $10^{-1}$ & 4.0133453 & 4.0072641 & 4.0037786 & 4.0134 \\
$10^{-2}$ & 6.9008759 & 6.9007779 & 6.9006943 & 6.9009 \\
$10^{-3}$ & 9.7942930 & 9.7942916 & 9.7942903 & 9.7942 \\
$10^{-6}$ & 18.474833 & 18.474833 & 18.474833 & - \\
$10^{-9}$ & 25.155374 & 25.155374 & 25.155374 & - \\
$10^{-12}$ & 35.835916 & 35.835916 & 35.835916 & - \\
\hline
\end{tabular}

Example 10. Calculate the self-inductance of the full disk coil of the radius $R_{1}=0.5 \mathrm{~m}$ and the number of turns $N=100$.

This work, Equation (11), gives

$$
L_{A-\text { full-disk }}=3.4847852 \mathrm{mH} \text {. }
$$

The same result is obtained in [31].

From [9] the self-inductance is

$$
L_{A-\text { Kajikawa }}=3.4848 \mathrm{mH} \text {. }
$$

\section{Conclusions}

The new accurate self-inductance formulas for the circular thick coils of a rectangular cross-section with radial and the azimuthal current densities are given. The formulas are obtained in the form of a single integral whose kernel function on the interval of integration is the sum of the elementary functions. The special cases of these formulas give the self-inductance for thin-disk coil and the thin-wall solenoid in the closed and semi-analytical form. For the asymptotic case, the self-inductance of the thin-wall solenoid with an extremely small height is developed for the first time in literature. Thus, all cases for circular coils with and without cross-section are given. The presented method can be helpful for engineers, physicists and people who work in this domain so that they can easily use all formulas in Mathematica or MATLAB programming.

Author Contributions: Conceptualization, S.B. and C.A.; methodology, S.B.; software, S.B.; validation, S.B.; formal analysis, C.A.; investigation, S.B and C.A..; resources, S.B.; data curation, S.B.; writing-original draft preparation, S.B.; writing-review and editing, S.B.; visualization, C.A.. All authors have read and agreed to the published version of the manuscript.

Funding: This research received no external funding.

Acknowledgments: The authors would like to thank J.T. Conway of the University of Agder, Grimstad, Norway and Y. Ren of High Magnetic Field Laboratory, Chinese Academy of Sciences, Hefei, China for providing extremely high precision calculations for the self-inductance calculation, which have proven invaluable in validating the method presented here.

Conflicts of Interest: The authors declare no conflict of interest

\section{Appendix A}

Previously, in Equation (2) the following changes are made to simplify the four integrations,

$r_{1}=x R_{1}, r_{2}=y R_{1}, z_{1}=v R_{1}, z_{2}=z R_{1}, t=v-z, l=b R_{1}, R_{2}=\alpha R_{1}, \theta=\pi-2 \beta$. The first integration over the variable $y$ in Equation (2) gives

$$
I_{1}=\int_{1}^{\alpha} \frac{d y}{R_{12}}=\operatorname{asinh} \frac{\alpha+x \cos (2 \beta)}{\sqrt{x^{2} \sin ^{2}(2 \beta)+t^{2}}}-\operatorname{asinh} \frac{1+x \cos (2 \beta)}{\sqrt{x^{2} \sin ^{2}(2 \beta)+t^{2}}} .
$$


The second integration over the variable $x$ in Equation (2) gives

$$
\begin{aligned}
I_{2}=\int_{1}^{\alpha} I_{1} d x=2[ & \alpha \operatorname{asinh} \frac{\alpha+\alpha \cos (2 \beta)}{\sqrt{\alpha^{2} \sin ^{2}(2 \beta)+t^{2}}}+\operatorname{asinh} \frac{1+\alpha \cos (2 \beta)}{\sqrt{\sin ^{2}(2 \beta)+t^{2}}} \\
& -\alpha \operatorname{asinh} \frac{1+\alpha \cos (2 \beta)}{\sqrt{\alpha^{2} \sin ^{2}(2 \beta)+t^{2}}}-\operatorname{asinh} \frac{1+\cos (2 \beta)}{\sqrt{\sin ^{2}(2 \beta)+t^{2}}} \\
& +\frac{t}{\sin (2 \beta)} \arctan \frac{\alpha \sin ^{2}(2 \beta)-t^{2} \cos (2 \beta)}{\operatorname{tin}(2 \beta) \sqrt{t^{2}+\alpha^{2}+2 \alpha \cos (2 \beta)+1}} \\
& -\frac{t}{2 \sin (2 \beta)} \arctan \frac{\alpha^{2} \sin ^{2}(2 \beta)-t^{2} \cos (2 \beta)}{\operatorname{tsin}(2 \beta) \sqrt{t^{2}+4 \alpha^{2} \cos ^{2}(\beta)}} \\
& \left.-\frac{t}{2 \sin (2 \beta)} \arctan \frac{\sin ^{2}(2 \beta)-t^{2} \cos (2 \beta)}{\operatorname{tin}(2 \beta) \sqrt{t^{2}+4 \cos ^{2}(\beta)}}\right] .
\end{aligned}
$$

The third integration over the variable $v$ in Equation (2) gives

$$
\begin{aligned}
& I_{3}=\int_{0}^{b} \frac{I_{2}}{2} d v=\left[\frac{t^{2}}{2 \sin (2 \beta)} \arctan \frac{\alpha \sin ^{2}(2 \beta)-t^{2} \cos (2 \beta)}{t \sin (2 \beta) \sqrt{t^{2}+\alpha^{2}+2 \alpha \cos (2 \beta)+1}}\right. \\
&-\frac{t^{2}}{4 \sin (2 \beta)} \arctan \frac{\alpha^{2} \sin ^{2}(2 \beta)-t^{2} \cos (2 \beta)}{t \sin ^{2}(2 \beta) \sqrt{t^{2}+4 \alpha^{2} \cos ^{2}(\beta)}} \\
&-\frac{t^{2}}{4 \sin (2 \beta)} \arctan \frac{\sin ^{2}(2 \beta)-t^{2} \cos ^{2}(2 \beta)}{t \sin (2 \beta) \sqrt{t^{2}+4 \cos ^{2}(\beta)}} \\
&-\frac{\left[\left(\alpha^{2}+1\right) \cos (2 \beta)+2 \alpha\right]}{2} \operatorname{asinh} \frac{t}{r_{0}} \\
&+\frac{\alpha^{2}[\cos (2 \beta)+1]}{2} \operatorname{asinh} \frac{t}{2 \alpha \cos (\beta)}+\frac{[\cos (2 \beta)+1]}{2} \operatorname{asinh} \frac{t}{2 \cos (\beta)} \\
&+\alpha \operatorname{tasinh} \frac{\alpha+\alpha \cos (2 \beta)}{\sqrt{\alpha^{2} \sin ^{2}(2 \beta)+t^{2}}}+\operatorname{tasinh} \frac{1+\alpha \cos (2 \beta)}{\sqrt{\sin ^{2}(2 \beta)+t^{2}}} \\
&-\alpha \operatorname{tasinh} \frac{1+\alpha \cos (2 \beta)}{\sqrt{\alpha^{2} \sin ^{2}(2 \beta)+t^{2}}}-\operatorname{tasinh} \frac{\alpha+\cos (2 \beta)}{\sqrt{\sin ^{2}(2 \beta)+t^{2}}} \\
&-\frac{\alpha^{2} \sin (2 \beta)}{2} \arctan \frac{t \cos (\beta)}{\sin (2 \beta) \sqrt{t^{2}+4 \alpha^{2} \cos ^{2}(\beta)}} \\
&-\frac{\sin (2 \beta)}{2} \arctan \frac{t \cos (\beta)}{\sin (2 \beta) \sqrt{t^{2}+4 \cos ^{2}(\beta)}} \\
&+\frac{\alpha^{2} \sin (2 \beta)}{2} \arctan \frac{t \cos ^{2}(\beta)}{\sin (2 \beta) \sqrt{t^{2}+4 \alpha^{2} \cos ^{2}(\beta)}} \\
&+\frac{\alpha^{2} \sin (2 \beta)}{2} \arctan \frac{t(1+\alpha \cos (\beta))}{\alpha \sin (2 \beta) \sqrt{t^{2}+\alpha^{2}+2 \alpha \cos (2 \beta)+1}} \\
&\left.+\frac{\sin (2 \beta)}{2} \arctan \frac{t\left(\alpha+\cos ^{2}(\beta)\right)}{\alpha \sin (2 \beta) \sqrt{t^{2}+\alpha^{2}+2 \alpha \cos (2 \beta)+1}}\right]-z \\
& b-z
\end{aligned}
$$

Finally, the fourth integration over the variable $z$ in Equation (2),

$$
\mathrm{I}_{4}=\int_{0}^{\mathrm{b}}\left(2 \mathrm{I}_{3}\right) \mathrm{dz}
$$

leads to $T_{n}, n=1,2, \ldots, 8$, which appears in expression (5) for the self-inductance $L_{R}$ of the circular coil with a rectangular cross-section and radial current density. 


\section{Appendix B}

Previously, in Equation (4) the following changes are made to simplify the four integrations, $r_{1}=x R_{1}, r_{2}=y R_{1}, z_{1}=v R_{1}, z_{2}=z R_{1}, t=v-z, l=b R_{1}, R_{2}=\alpha R_{1}, \theta=\pi-2 \beta$. The first integration over the variable $y$ in Equation (4) gives

$$
\begin{aligned}
& I_{1}=\int_{1}^{\alpha} \frac{y d y}{R_{12}}=\left[\sqrt{x^{2}+2 x \alpha \cos (2 \beta)+\alpha^{2}+t^{2}}-\sqrt{x^{2}+2 x \cos (2 \beta)+1+t^{2}}\right. \\
& \left.-x \cos (2 \alpha) \frac{\alpha+x \cos (2 \beta)}{\sqrt{x^{2} \sin ^{2}(2 \beta)+t^{2}}}+x \cos (2 \alpha) \frac{1+x \cos (2 \beta)}{\sqrt{x^{2} \sin ^{2}(2 \beta)+t^{2}}}\right] .
\end{aligned}
$$

The second integration over the variable $x$ in Equation (4) gives

$$
\begin{aligned}
I_{2}=\int_{1}^{\alpha} x I_{1} d x= & \frac{2}{3}\left[-\alpha^{3} \cos (2 \beta) \operatorname{asinh} \frac{\alpha+\alpha \cos (2 \beta)}{\sqrt{\alpha^{2} \sin ^{2}(2 \beta)+t^{2}}}\right. \\
& -\cos (2 \beta) \operatorname{asinh} \frac{1+\cos (2 \beta)}{\sqrt{\sin ^{2}(2 \beta)+t^{2}}} \\
& +\alpha^{3} \cos (2 \beta) \operatorname{asinh} \frac{1+\alpha \cos (2 \beta)}{\sqrt{\alpha^{2} \sin ^{2}(2 \beta)+t^{2}}} \\
& +\cos (2 \beta) \operatorname{asinh} \frac{\alpha+\cos (2 \beta)}{\sqrt{\sin ^{2}(2 \beta)+t^{2}}} \\
& +\left[\alpha^{2}+\frac{t^{2}}{2 \sin ^{2}(2 \beta)}\right] \sqrt{4 \alpha^{2} \cos ^{2}(\beta)+t^{2}} \\
& +\left[1+\frac{t^{2}}{2 \sin ^{2}(2 \beta)}\right] \sqrt{4 \cos ^{2}(\beta)+t^{2}} \\
& -\left[\alpha^{2}+1+\frac{t^{2}}{\sin ^{2}(2 \beta)}\right] \sqrt{t^{2}+\alpha^{2}+2 \alpha \cos (2 \beta)+1} \\
& +\frac{t^{3} \cos (2 \beta)}{\sin ^{3}(2 \beta)} \arctan \frac{\alpha \sin ^{2}(2 \beta)-t^{2} \cos (2 \beta)}{\operatorname{tin}(2 \beta) \sqrt{t^{2}+\alpha^{2}+2 \alpha \cos (2 \beta)+1}} \\
& -\frac{t^{3} \cos (2 \beta)}{2 \sin ^{3}(2 \beta)} \arctan \frac{\alpha^{2} \sin ^{2}(2 \beta)-t^{2} \cos (2 \beta)}{\operatorname{tin}(2 \beta) \sqrt{4 \alpha^{2} \cos ^{2}(\beta)+t^{2}}} \\
& \left.-\frac{t^{3} \cos (2 \beta)}{2 \sin ^{3}(2 \beta)} \arctan \frac{\sin ^{2}(2 \beta)-t^{2} \cos (2 \beta)}{\operatorname{tin}(2 \beta) \sqrt{4 \cos ^{2}(\beta)+t^{2}}}\right]
\end{aligned}
$$


The third integration over the variable $v$ in Equation (4) gives

$$
\begin{aligned}
I_{3}=-8 t \cos (2 \beta) & \operatorname{asinh} \frac{1+\cos (2 \beta)}{\sqrt{\sin ^{2}(2 \beta)+t^{2}}}+8 t \alpha^{3} \cos (2 \beta) \operatorname{asinh} \frac{1+\alpha \cos (2 \beta)}{\sqrt{\alpha^{2} \sin ^{2}(2 \beta)+t^{2}}} \\
& +8 t \cos (2 \beta) \operatorname{asinh} \frac{\alpha+\cos (2 \beta)}{\sqrt{\sin ^{2}(2 \beta)+t^{2}}} \\
& -\frac{t^{4} \cos (2 \beta)}{\sin ^{3}(2 \beta)} \arctan \frac{\alpha^{2} \sin ^{2}(2 \beta)-t^{2} \cos (2 \beta)}{\operatorname{tsin}(2 \beta) \sqrt{4 \alpha^{2} \cos ^{2}(\beta)+t^{2}}} \\
& -\frac{t^{4} \cos (2 \beta)}{\sin ^{3}(2 \beta)} \arctan \frac{\sin ^{2}(2 \beta)-t^{2} \cos (2 \beta)}{\operatorname{tsin}(2 \beta) \sqrt{4 \cos ^{2}(\beta)+t^{2}}} \\
& +\frac{2 t^{4} \cos (2 \beta)}{\sin ^{3}(2 \beta)} \arctan \frac{\alpha \sin ^{2}(2 \beta)-t^{2} \cos (2 \beta)}{\operatorname{tsin}(2 \beta) \sqrt{t^{2}+\alpha^{2}+2 \alpha \cos (2 \beta)+1}} \\
& +6 \alpha^{2} \sin ^{2}(2 \beta) \operatorname{asinh} \frac{t}{2 \alpha \cos (\beta)}+6 \sin ^{2}(2 \beta) \operatorname{asinh} \frac{t}{2 \cos (\beta)} \\
& +3\left[2\left(\alpha^{4}+1\right) \cos (2 \beta)-\left(\alpha^{2}+1\right)^{2}\right] \operatorname{asinh} \frac{t}{r_{0}} \\
& +6 \alpha^{4} \sin (2 \beta) \cos (2 \beta) \arctan \frac{t \cos (\beta)}{\sin (2 \beta) \sqrt{t^{2}+4 \alpha^{2} \cos { }^{2}(\beta)}} \\
& +6 \sin (2 \beta) \cos (2 \beta) \arctan \frac{t \cos (\beta)}{\sin (2 \beta) \sqrt{t^{2}+4 \cos ^{2}(\beta)}} \\
& -6 \alpha^{4} \sin (2 \beta) \cos (2 \beta) \arctan \frac{t(1+\alpha \cos (\beta))}{\alpha \sin (2 \beta) \sqrt{t^{2}+\alpha^{2}+2 \alpha \cos (2 \beta)+1}} \\
& -6 \sin (2 \beta) \cos (2 \beta) \arctan \frac{t(\alpha+\cos (\beta))}{\sin (2 \beta) \sqrt{t^{2}+\alpha^{2}+2 \alpha \cos (2 \beta)+1}} \\
& +t\left[5 \alpha^{2}+\frac{t^{2}}{\sin ^{2}(2 \beta)}\right] \sqrt{4 \alpha^{2} \cos { }^{2}(\beta)+t^{2}} \\
+ & t\left[5+\frac{t^{2}}{\sin ^{2}(2 \beta)}\right] \sqrt{4 \cos ^{2}(\beta)+t^{2}} \\
& \left.-t\left[5\left(\alpha^{2}+1\right)+\frac{2 t^{2}}{\sin ^{2}(2 \beta)}\right] \sqrt{t^{2}+\alpha^{2}+2 \alpha \cos (2 \beta)+1}\right]
\end{aligned}
$$

Finally, the fourth integration over the variable $z$ in Equation (4),

$$
I_{4}=\int_{0}^{b} I_{3} d z
$$

leads to $S_{n}, n=1,2, \ldots, 11$, which appears in expression (7) for the self-inductance $L_{A}$ of a circular coil with a rectangular cross-section and azimuthal current density.

\section{References}

1. Grover, F.W. Inductance Calculations; Dover: New York, NY, USA, 1964.

2. Dwight, H.B. Electrical Coils and Conductors; McGraw-Hill Book Company, Inc.: New York, NY, USA, 1945.

3. Snow, C. Formulas for Computing Capacitance and Inductance; National Bureau of Standards Circular 544: Washington, DC, USA, December 1954.

4. Spielrein, J. Die Induktivität eisenfreier Kreisringspulen. Archiv Elektrotechnik. 1915, 3, 187-202. [CrossRef]

5. Rosa, E.B.; Grover, F.W. Scientific Papers of the National Bureau of Standards, 3rd ed.; National Bureau of Standards: Washington, DC, USA, 1948.

6. Kalantarov, P.L. Inductance Calculations; National Power Press: Moscow, Russia, 1955.

7. Watson, G.N. A Treaties on the Theory of Bessel Functions, 2nd ed.; Cambridge Univ. Press: Cambridge, $\mathrm{UK}, 1944$.

8. Yu, D.; Han, K.S. Self-inductance of air-core circular coils with rectangular cross section. IEEE Trans. Magn. 1987, 23, 3916-3921. [CrossRef] 
9. Kajikawa, K.; Kaiho, K. Usable Ranges of Some Expressions for Calculation of the Self-Inductance of a Circular Coil of Rectangular Cross Section. J. Cryog. Soc. Jpn. 1995, 30, 324-332. (In Japanese) [CrossRef]

10. Conway, J.T. Inductance Calculations for Circular Coils of Rectangular Cross Section and Parallel Axes Using Bessel and Struve Functions. IEEE Trans. Magn. 2009, 46, 75-81. [CrossRef]

11. Babic, S.; Akyel, C. Improvement in calculation of the self- and mutual inductance of thin-wall solenoids and disk coils. IEEE Trans. Magn. 2000, 36, 1970-1975. [CrossRef]

12. Conway, J.T. Exact solutions for the mutual inductance of circular coils and elliptic coils. IEEE Trans. Magn. 2011, 48, 81-94. [CrossRef]

13. Luo, Y.; Chen, B. Improvement of Self-Inductance Calculations for Circular Coils of Rectangular Cross Section. IEEE Trans. Magn. 2012, 49, 1249-1255. [CrossRef]

14. Luo, Y.; Wang, X.; Zhou, X. Inductance Calculations for Circular Coils With Rectangular Cross Section and Parallel Axes Using Inverse Mellin Transform and Generalized Hypergeometric Functions. IEEE Trans. Power Electron. 2016, 32, 1367-1374. [CrossRef]

15. Pankrac, V. Generalization of Relations for Calculating the Mutual Inductance of Coaxial Coils in Terms of Their Applicability to Non-Coaxial Coils. IEEE Trans. Magn. 2011, 47, 4552-4563. [CrossRef]

16. Liang, S.; Fang, Y. Analysis of Inductance Calculation of Coaxial Circular Coils With Rectangular Cross Section Using Inverse Hyperbolic Functions. IEEE Trans. Appl. Supercond. 2015, 25, 1-9. [CrossRef]

17. Župan, T.; Štih, Ž.; Trkulja, B. Fast and Precise Method for Inductance Calculation of Coaxial Circular Coils with Rectangular Cross Section Using the One-Dimensional Integration of Elementary Functions Applicable to Superconducting Magnets. IEEE Trans. Appl. Supercond. 2014, 24, 81-89. [CrossRef]

18. Dolezel, I. Self-inductance of an air cylindrical coil. Acta Tech. CSAV 1989, 34, 443-473.

19. Bitter, F. The Design of Powerful Electromagnets Part II. The Magnetizing Coil. Rev. Sci. Instrum. 1936, 7, 482-489. [CrossRef]

20. Conway, J.T. Non coaxial force and inductance calculations for bitter coils and coils with uniform radial current distributions. In Proceedings of the 2011 International Conference on Applied Superconductivity and Electromagnetic Devices, Sydney, NSW, Australia, 14-16 December 2011; pp. 61-64.

21. Ren, Y.; Wang, F.; Kuang, G.; Chen, W.; Tan, Y.; Zhu, J.; He, P. Mutual Inductance and Force Calculations between Coaxial Bitter Coils and Superconducting Coils with Rectangular Cross Section. J. Supercond. Nov. Magn. 2010, 24, 1687-1691. [CrossRef]

22. Ren, Y.; Kuang, G.; Chen, W. Inductance of Bitter Coil with Rectangular Cross-section. J. Supercond. Nov. Magn. 2012, 26, 2159-2163. [CrossRef]

23. Babic, S.; Akyel, C. Mutual inductance and magnetic force calculations for coaxial bitter disk coils (Pancakes). IET Sci. Meas. Technol. 2016, 10, 972-976. [CrossRef]

24. Babic, S.; Akyel, C. Calculation of mutual inductance and magnetic force between two thick coaxial Bitter coils of rectangular cross section. IET Electr. Power Appl. 2017, 11, 441-446. [CrossRef]

25. Filanovsky, I. On Design of $60 \mathrm{GHz}$ Solid-State Transformers Modeled as Coupled Bitter Coils. In Proceedings of the 2019 IEEE 62nd International Midwest Symposium on Circuits and Systems (MWSCAS), Dallas, TX, USA, 4-7 August 2019; pp. 1021-1024.

26. Babic, S.I.; Akyel, C. An improvement in the calculation of the thin disk coils with air-core. WSEAS Trans. Circuits Syst. 2004, 3, 1621-1626.

27. Babic, S.; Akyel, C.; Erdogan, L.; Babic, B. The Influence of the Numerical Integration with MATLAB and Mathematica Programming in the Treatment of the Logarithmic Singularity of the Inductance of the Pancake. Am. J. Math. Comput. Sci. 2016, 1, 62-66.

28. Gradshteyn, S.; Ryzhik, I.M. Table of Integrals, Series and Products; Academic Press Inc.: New York, NY, USA; London, UK, 1965.

29. Abramowitz, M.; Stegun, I.S. Handbook of Mathematical Functions; Dover: New York, NY, USA, 1972.

30. Brychkov, Y.A. Handbook of Special Functions: Derivatives, Integrals, Series and Other Formulas; CRC Press: Boca Raton, FL, USA, 2008.

31. Conway, J.T. Analytical Solutions for the Self- and Mutual Inductances of Concentric Coplanar Disk Coils. IEEE Trans. Magn. 2012, 49, 1135-1142. [CrossRef] 
32. Babic, S.; Akyel, C.; Boudjada, N. The Simplest Formulas for Self-Inductance, Mutual Inductance and Magnetic Force of Coaxial Cylindrical Magnets and Thin Coils; Recent Researches in Circuits and Systems; WSEAS: Kos, Greece, 2012.

33. Digital Library of Mathematical Function (Elliptic Integrals, Asymptotic Approximations), NIST, National Institute of Standard and Technology. Available online: https://dlmf.nist.gov/19.12 (accessed on 2 June 2020).

(C) 2020 by the authors. Licensee MDPI, Basel, Switzerland. This article is an open access article distributed under the terms and conditions of the Creative Commons Attribution (CC BY) license (http://creativecommons.org/licenses/by/4.0/). 